\title{
Dispersion management in nonlinear photonic crystal fibres with nanostructured core
}

\author{
Ryszard Buczynski \\ rbuczyns@igf.fuw.edu.pl \\ Dariusz Pysz
}

Ryszard Stepien

Rafal Kasztelanic

Ireneusz Kujawa

Marcin Franczyk

\section{Adam Filipkowski}

\author{
Andrew J. Waddie
}

Mohammad R. Taghizadeh

\author{
Institute of Electronic Materials Technology, Wólczyńska 133, 01-919 Warsaw, Poland \\ Institute of Electronic Materials Technology, Wólczyńska 133, 01-919 Warsaw, Poland \\ Institute of Electronic Materials Technology, Wólczyńska 133, 01-919 Warsaw, Poland \\ University of Warsaw, Faculty of Physics, 02-093 Warsaw, Poland
}

Institute of Electronic Materials Technology, Wólczyńska 133, 01-919 Warsaw, Poland

Institute of Electronic Materials Technology, Wólczyńska 133, 01-919 Warsaw, Poland

Institute of Electronic Materials Technology, Wólczyńska 133, 01-919 Warsaw, Poland

Heriot-Watt University, School of Engineering and Physical Sciences, Edinburgh EH14 4AS, Scotland, UK

Heriot-Watt University, School of Engineering and Physical Sciences, Edinburgh EH14 4AS, Scotland, UK

The subwavelength structure of the core of a photonic crystal fibre can modify its dispersion characteristic and significantly shift the zero dispersion wavelength. The dispersion properties of photonic crystal fibres with core structures made of a $2 \mathrm{D}$ lattice of subwavelength air holes and various glass inclusions are studied. We show that a modification of the core structure can give flat dispersion over a range of over $300 \mathrm{~nm}$ and can shift the zero dispersion wavelength over $700 \mathrm{~nm}$ while the core diameter and photonic cladding remain unchanged. The developed photonic crystal fibre with nanorod core has successfully demonstrated supercontinuum generation in NIR. [DOI: $10.2971 /$ jeos.2011.11038]

Keywords: photonic crystal fibers, supercontinuum generation, dispersion, soft glass

\section{INTRODUCTION}

Photonic crystal fibres (PCFs) are a very efficient medium for supercontinuum generation [1]. The manipulation of the photonic cladding parameters allows tailoring of the dispersion properties of the fibre. For silica-based photonic crystal fibre the zero dispersion wavelength (ZDW) can be shifted into the visible region and a range of pulsed sources can be efficiently used for broadband supercontinuum generation covering the entirety of the visible and near infrared spectrum.

Recently a lot of interest has focused on nonlinear PCFs made of tellurite and heavy metal oxide glasses, because these materials offer much higher nonlinear coefficients than silica glasses and more broadband transmission into the mid IR range [2]. However the dispersion properties of the fibres with simple photonic cladding prevent the use of efficient pump sources since the ZDW is placed in the IR region beyond 1.6 $\mu \mathrm{m}$. A reduction of the core diameter allows a shift of the ZDW to shorter wavelengths, but simultaneously the attenuation of the fibre and coupling losses increase dramatically.

Recently the possibility of the manipulation of the fibre prop- erties by structuring of the core has been developed for sensor applications [3,4]. A multiply highly doped area in the core was used to enhance the nonlinearity of a photonic crystal fibre and permit the efficient broadening of the supercontinuum spectrum [5]. The concept of engineering of dispersion properties in PCFs with subwavelength single air holes in core was introduced and modeled by Saitoh et al. [6]. tailoring zero dispersion and flat dispersion achevment was presented. Kibler et al. shown that introduction of subwavelength single air holes in core modify significatly dispersion properties of the PCF and it can be used for spectral tunelling of solitons [7]. Successful development of this type of fibers are reported by Wiederhecker at al [8], Ruan at al. [9] and Liu at al. [10]. In these cases a modification group velocity dispersion is also observed. The works [8,9] focus on a possibility of field enhancement in the subwavelength air core, while Liu at al. shows that structuring a core of PCF may lead to a stable soliton self-frequency shift and subpetahertz sideband generation through four-wave mixing [10]. Recently the concept of a fibre with an array of microporous air-suspended core PCF based on chalcogenide glasses was introduced and modeled 
by Ung and Skorobogatiy [11]. The possibility of a red-shift of the ZDW to $10.5 \mu \mathrm{m}$ was shown.

In this paper we propose to structure a core of the PCF with high index inclusions to tailor its dispersion properties and blue shift of zero dispersion wavelength. In this case the core of a standard PCF with air-hole cladding is structured with a single subwavelength rods.

\section{Design of photonic crystal fibres with nanostructured core}

We consider in the simulations various pairs of two thermally matched glasses (NC-21, SF6 and F2 air glass) to build subwavelength structures in the core. The structures with a high and low contrast are modelled. The modal and dispersion properties of the PCFs are calculated by means of a finite element method (FEM) using the COMSOL Multiphysics software [12]. We study the influence of core structure on the fibre dispersion in terms of the nano-sized rod diameter and their distribution. In addition, the simulation takes into account the material dispersion of the considered glasses through the Sellmeier coefficients [13].

To determine the character of the dispersion modification, we have performed indicative simulations. In the simulations we compare the dispersion properties of PCFs with air and high refractive index inclusions for a selected structure with the lattice constant $\Lambda=2.0 \mathrm{~mm}$ and filling factor $f=0.95$. We assume the presence of a subwavelength $2 \mathrm{D}$ regular hexagonal structure composed of a $7 \times 7$ array. The subwavelength structure has the lattice constant $\Lambda_{c}=300 \mathrm{~nm}$ with inclusions of the diameter of $d c=100 \mathrm{~nm}$. As a background glass, the low index borosilicate glass NC21 synthesised in-house at ITME is used. A heavy metal oxide glass (SF6) and air are used as subwavelength inclusions in the core. Simulation results show that the structure of the core has a strong influence on the dispersion characteristics (Fig. 1).

It might be unexpected to see that the red shift of the ZDW is observed for both cases high and low inclusions with respect to a similar structure of PCF with a solid core. However the origin of the red shift of the ZDW is different in both cases. For the air hole nanostructure, this behaviour is related to the lower effective refractive index of the core. Simmilar behaviour was previously reported in case of singe air hole inclusion [6, 8]. Consequently for high index inclusions we would expect a blue shift of the ZDW. However this effect compensated for by the material dispersion properties of the high index glass. Since the ZDW for bulky heavy metal nonlinear glasses is beyond $1.9 \mu \mathrm{m}$ this effect is dominant and a red shift of the ZDW is observed.

Although a successful development of PCF with a single nanohole was previously reported [8]-[10], the development of PCFs with a regular lattice of subwavelength air holes in the core still remains a challenge for fiber technology [14]. Therefore an interesting alternative approach seems to be the use of subwavelength high index glass inclusions in the core. An additional advantage of this approach is an increase of the glass
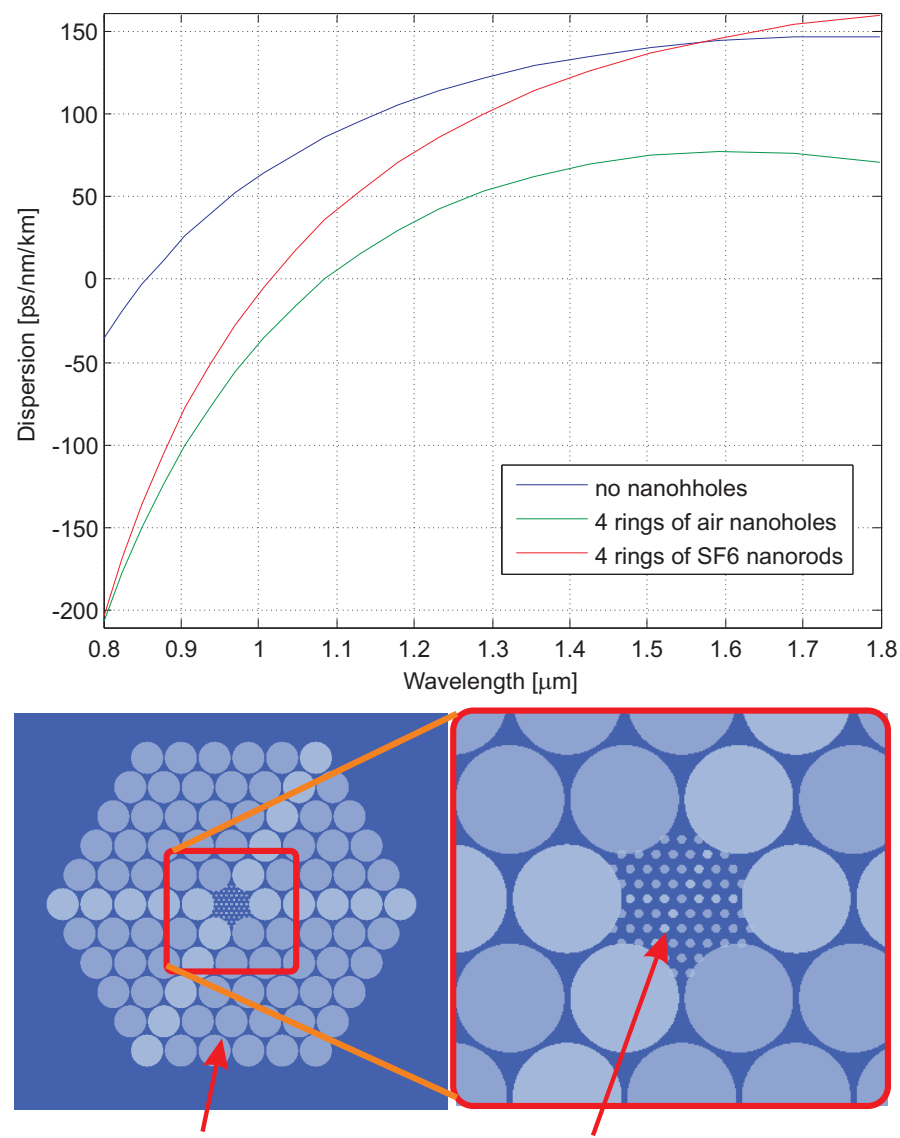

air holes

air holes or glass inclusions

FIG. 1 Dispersion properties of the PCFs with the nanostructured core with $\Lambda_{c}=300$ $\mathrm{nm} d c=100 \mathrm{~nm}$. The photonic cladding is composed of air holes ordered in a hexagonal lattice with $\Lambda=2.0 \mu \mathrm{m}, d=1.9 \mu \mathrm{m}$, A silicate glass $\mathrm{NC}_{21}$ is used as the substrate glass. Core subwavelength structure: air nanoholes or SF6 nanorods. Schematic of photonic crystal fibre with nanostructured core. The photonic cladding is composed of a regular lattice of air holes, while the core consists of subwavelength air holes or various glass inclusions.

nonlinearity in the core which influences the efficiency of the nonlinear phenomena in the fibre. The introduction of a subwavelength structure results in a red shift of the ZDW (Fig. 2). It is important to note that the effective mode area of the considered fundamental modes in the successive structures depends on the number of rings. Therefore structures with a single high index inclusion and with a standard solid core might have similar dispersion properties but very different effective mode areas.

In case of a single subwavelength inclusion of high index glass in the core, the fibre dispersion properties will vary with the diameter of the inclusion. A decrease of the inclusion diameter results in a blue shit of the ZDW for fundamental modes (Fig. 3). On the other hand a increase of the inclusion cause decrease of effective mode area of the fundamental mode (Fig. 4).

It is important to note, that the excitation of the fundamental mode with a very small effective mode area is of low efficiency and therefore the second mode will play a dominant role in the energy transfer and will determine the dispersion properties of the fibre. The modal and dispersion properties of this second mode are very different from those of the fundamen- 


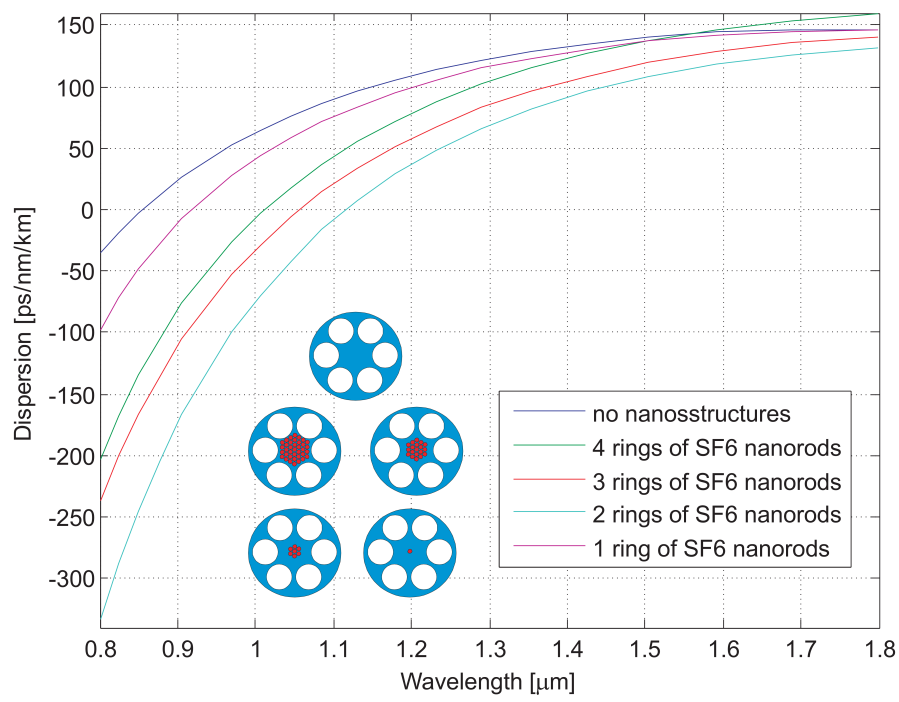

FIG. 2 Dispersion properties of the PCFs with the lattice of the subwavelength SF6 glass inclusions in the core with various number of rings. The photonic cladding is composed of air holes ordered in hexagonal lattice with $\Lambda=2.0 \mu \mathrm{m}, d=1.9 \mu \mathrm{m}$, The lattice pitch of subwavelength structure in the core is $\Lambda_{c}=300 \mathrm{~nm}$, and SF6 nanorod diameter $d c=160 \mathrm{~nm}$. A silicate glass $\mathrm{NC} 21$ is used as a substrate glass.

tal mode. Appropriate selection of the diameter of the high index nanorod in the core allows us to neglect the fundamental mode and to optimise the dispersion properties of the second mode which plays a dominant role in the fibre. For a PCF with an SF6 nanorod with $400 \mathrm{~nm}$ diameter, the effective mode area of the fundamental mode is as small as $0.39 \mu \mathrm{m}^{2}$, while the effective mode area of the second mode is much larger at 2.94 $\mu \mathrm{m}^{2}$ (Fig. 5). Moreover the dispersion properties of the second mode allows access to anomalous dispersion in the range $790-1860 \mathrm{~nm}$.

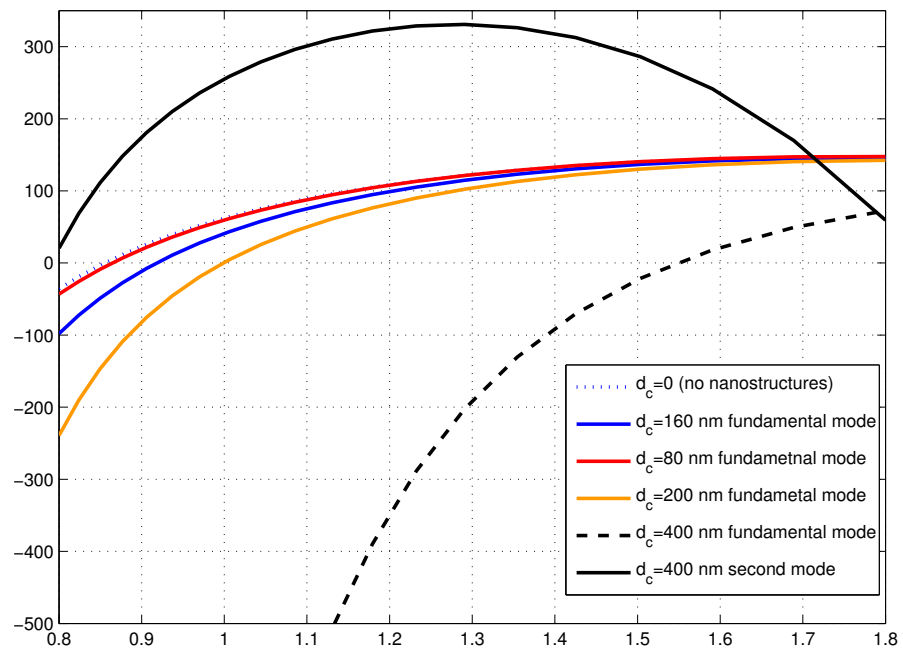

FIC. 3 Dispersion properties of the PCFs with the lattice of the subwavelength SF6 glass inclusions in the core with various diameters of inclusion. The photonic cladding is composed of air holes ordered in hexagonal lattice with $\Lambda=2.0 \mu \mathrm{m}, d=1.9 \mu \mathrm{m}$. A silicate glass $\mathrm{NC} 21$ is used as a substrate glass. As a reference dispersion curvature for solid core PCFs made of $\mathrm{NC}_{21}$ and SF6 glasses with similar photonic cladding parameters are shown.

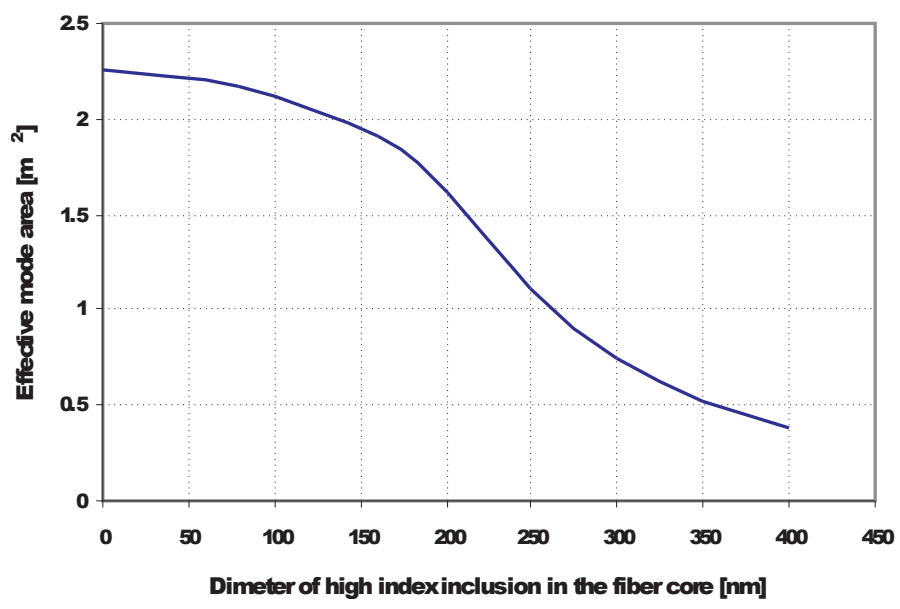

FIG. 4 Effective mode are of the fundamental mode in PCF with a single SF6 inclusion for various diameters of the inclusion for $800 \mathrm{~nm}$ wavelengths.

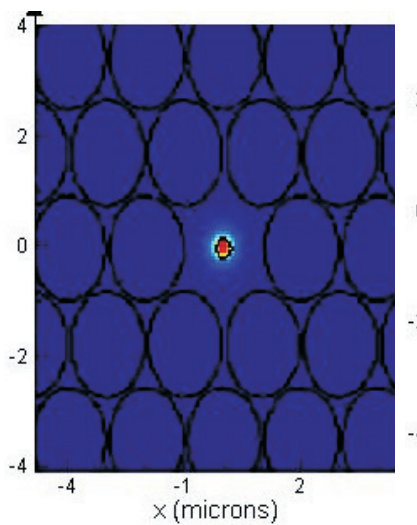

(a)

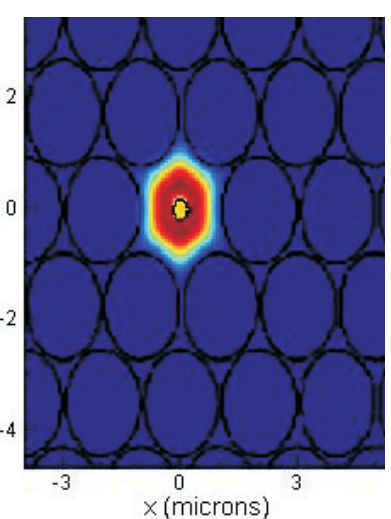

(b)
FIG. 5 Field distribution in the fundamental (a) and second (b) mode in PCF with a single SF6 inclusion (400 nm diameter) at the centre of the core. The photonic cladding is composed of air holes ordered in a hexagonal lattice with $\Lambda=2.0 \mu \mathrm{m}, d=1.9$ $\mu \mathrm{m}$. A silicate glass $\mathrm{NC} 21$ is used as a substrate glass.

\section{Development and characterization of photonic crystal fibre with nanostructured core}

Based on the performed simulations we have recently developed a PCF with the high index SF6 inclusion in the core. The fibre is made of silicate glass NC21 (55\% SiO2, 1\%Al2O3, 26\% B2O3, 3\% Li2O, 9.5\% Na2O, 5.5\% K2O, 0.8\% As2O3) synthesized in-house with refractive index of $n_{D}=1.518$. Nonlinear Kerr refractive index of NC21 glass is close to that of pure silica and has been measured as $1.1 \times 10^{-20} \mathrm{~m}^{2} / \mathrm{W}$ [13]. The fabricated fibre is composed of seven rings of holes ordered in a hexagonal lattice with the filling factor of $d / \Lambda=0.91$ and the lattice constant of $\Lambda=2.2 \mu \mathrm{m}$ (Fig. 6). The core is created by replacement of the central capillary with tubes made of similar glass NC21 with a SF6 rod. The core has a diameter of 2.2 $\mu \mathrm{m}$ while the SF6 nanorod has a diameter of $450 \mathrm{~nm}$.

The parameters of the developed PCF are slightly different those of the modelled one in previous chapter. Therefore modal and dispersion properties were simulated based on SEM photo taking into account all fabrication imperfections (Fig. 7). We have identified that ZDWs for fundamental mode located in the high index subwavelength inclusion are placed at 1763 and $1773 \mathrm{~nm}$ for orthogonal polarization components, 


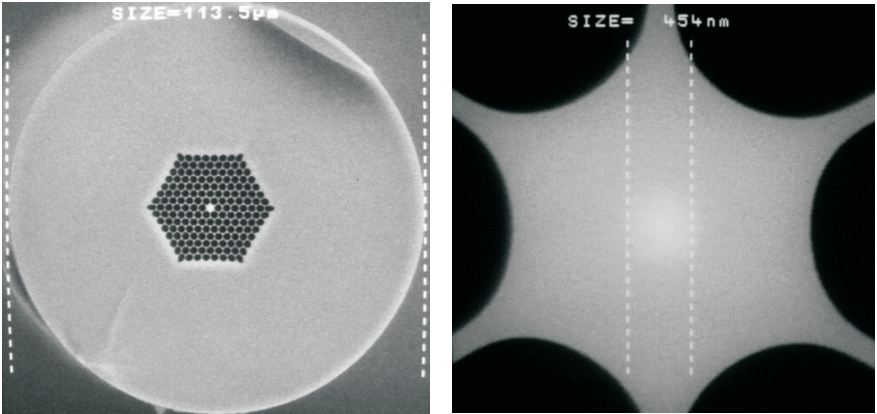

FIG. 6 A SEM photo of the nonlinear PCF with subwavelength glass structure in the core

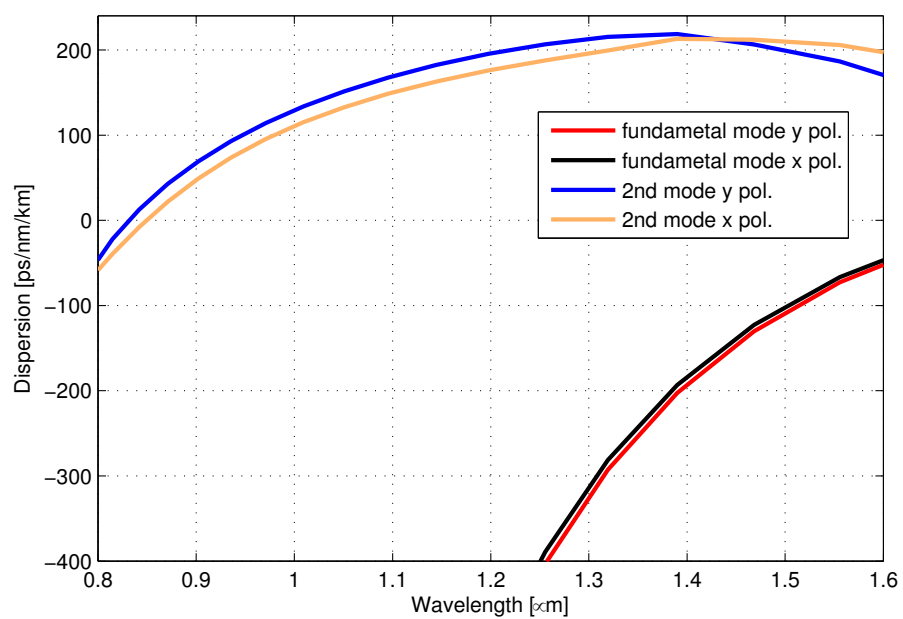

FIG. 7 Dispersion properties of the developed PCFs with subwavelength glass structure in the core.

respectively. Effective mode area is $0.38 \mu \mathrm{m}^{2}$. ZDWs for polarization components of second mode are $832 \mathrm{~nm}$ and $849 \mathrm{~nm}$, respectively. Effective mode area for this mode is $3.77 \mu \mathrm{m}^{2}$. Nonlinear coefficient for the fiber at pump wavelength 807 $\mathrm{nm}$ is $\gamma=23.1 \mathrm{~W}^{-1} \mathrm{~km}^{-1}$. However if we take into account a nanostructured character of the core we should use a modified nonlinear coefficient proposed by Afshar [15]. In this case nonlinear coefficient is higher $\gamma_{V}=28.5 \mathrm{~W}^{-1} \mathrm{~km}^{-1}$.

We have performed supercontinuum generation with a low power femtosecond oscillator (Fig. 8). As a pump we have used a Ti:Sapphire laser with a pulse width of $100 \mathrm{fs}$ and pulse energy up to $2.6 \mathrm{~nJ}$ at $807 \mathrm{~nm}$. The coupling efficiency between the laser beam and the measured fibre is $35 \%$. Experimentally, supercontinuum generation in the range $6651055 \mathrm{~nm}$ with flatness below $12 \mathrm{~dB}$ has been achieved in a $14 \mathrm{~cm}$ long section of PCF for $2.6 \mathrm{~nJ}$ input pulse energy. The generated spectrum for various pulse energies is shown in Fig. 9.

The obtained spectra shows typical behaviour of fiber

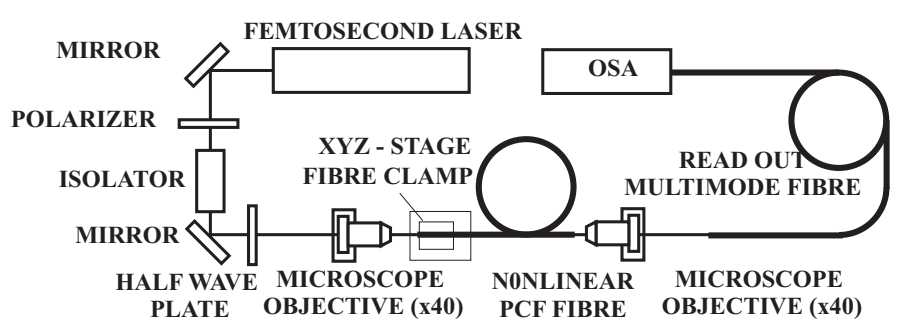

FIG. 8 A scheme of experimental setup

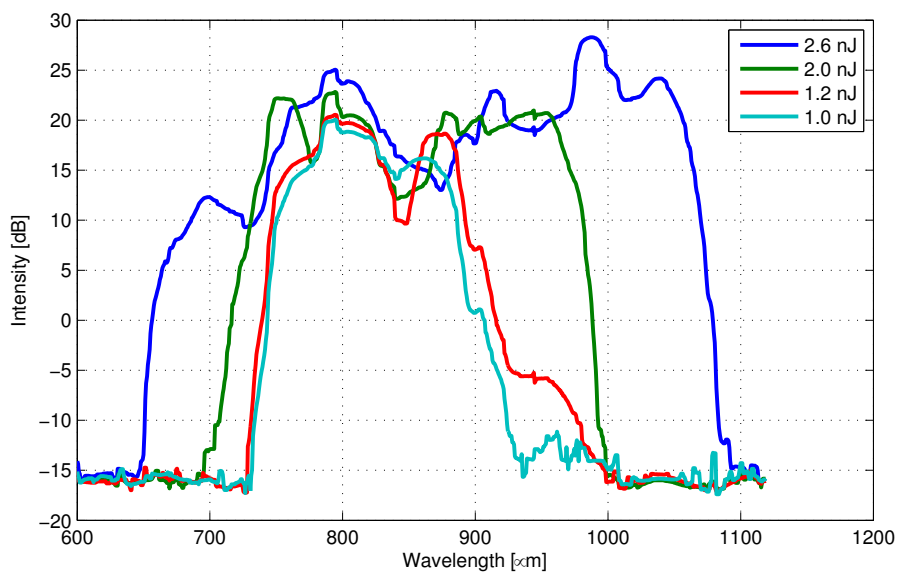

FIG. 9 Supercontinuum generation in the $14 \mathrm{~cm}$ long PCF with subwavelength glass structure in the core in the function of input pulse energy

pumped in anomalous dispersion regime. However at the beginning self phase modulation is responsible for peak broadening since pump wavelength is near ZWD of the fiber at the normal dispersion side. With increased of wavelength a soliton can formed in proximity to the pump at anomalous dispersions wavelength. For low input power second peak is observed at longer wavelength with respect to pump wavelength. A red shift of this peak with increasing power can be observed until $990 \mathrm{~nm}$ when pulse energy is increased. This phenomena can be explained with soliton-self frequency shift (SSFS).

As the input power is further increased up to $2.6 \mathrm{~nJ}$ of energy pulse a red-shifted soliton brunch with intensity peak at 990 generates blue-shifted dispersion wave with maximum peak at $700 \mathrm{~nm}$. In order to find out whether the dispersion wave are responsible for generation of spectra in short and long wavelengths range we have modelled phase matching conditions between hypothetic soliton and dispersion wave in the fiber according to equation [10];

$$
\delta \beta=\beta(\omega)-\beta\left(\omega_{s}\right)=\sum_{m=2}^{M} \frac{\beta_{m}\left(\omega-\omega_{s}\right)}{2 !}-\frac{\gamma P}{2}
$$

where $\beta_{m}$ coefficients denotes the $\mathrm{m}^{\text {th }}$ order derivative of $\beta(\omega)$, calculated at pump wavelength $\omega_{s}, \beta_{m}=\delta^{m} \beta /\left.\delta \omega^{m}\right|_{\omega_{s}}$, $\gamma$ is the fiber nonlinear coefficient and $P$ is the soliton peak power. The calculated $\beta_{m}$ values of the expansion for the considered mode at $\lambda_{0}=807 \mathrm{~nm}$. Phase matching between 990 $\mathrm{nm}$ soliton peak and $696 \mathrm{~nm}$ peak for the developed fiber is shown in Fig. 10. The existence of the soliton shows the anomalous dispersion of excited modes and proves the efficient coupling into the second mode.

Further increases in the spectrum are limited by the low energy of the input pulse. On the other hand use of longer fiber doesn't bring further improvement of the spectrum due to high attenuation of the fiber.

We have verified obtained results solving numerically a nonlinear Schroedinger equation (NLSE) for the considered fiber and similar experimental conditions for input pulses of $2.6 \mathrm{~nJ}$ with Split Step Fourier Method implemented by Travers et. al. 

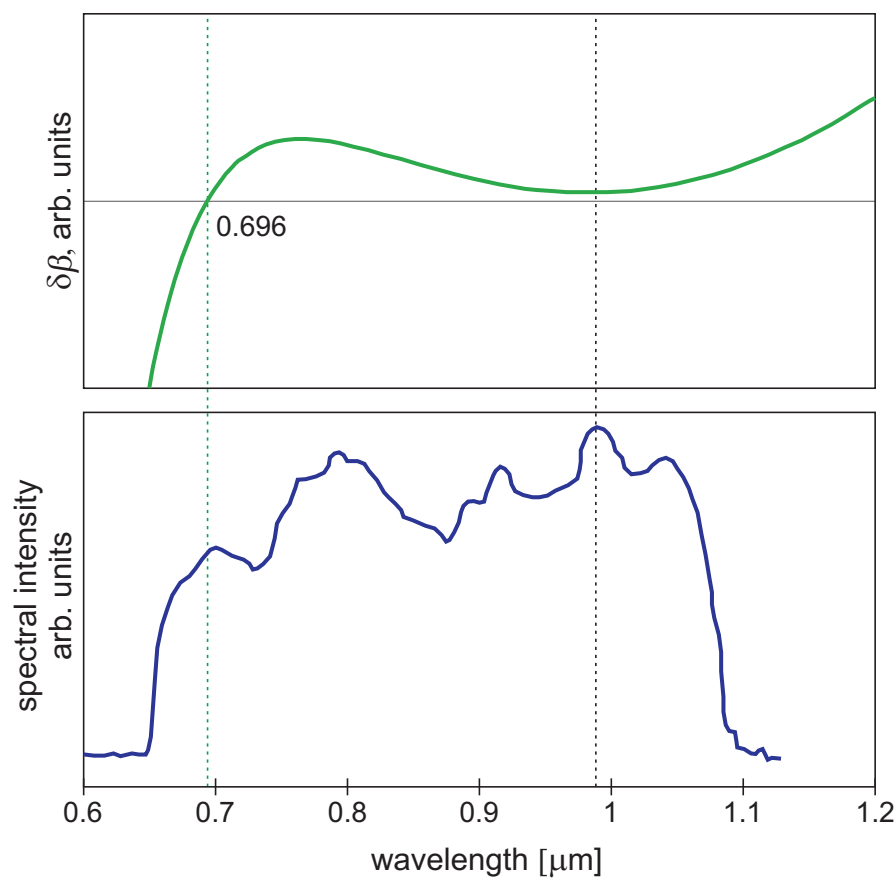

FIC. 10 Phase mismatch between soliton centred at $990 \mathrm{~nm}$ and blue shifted dispersive wave centred at $696 \mathrm{~nm}$. Dashed vertical lines shows the phase-matching wavelengths for blue and redshifted dispersive waves at experimental output spectra (lower panel)

[16]. We assumed in this case propagation of dominant second mode with $\mathrm{Y}$ polarization. Very similar results are obtained if the second mode with $X$ polarization is considered. Based on the simulation results, we obtain a slightly narrower spectrum in the range 660-960 nm (Fig. 11). In general, the simulated spectra are in good agreement with the experimental results. The differences between the spectra are due to the contributions of higher order modes to the experimental spectra. Furthermore, the fundamental mode can provide an increased contribution due to the increasing effective mode area with wavelength (Fig. 4) and hence, energy transfer between the second and fundamental modes.

The simulations show spectral features with a maximum intensity at $670 \mathrm{~nm}$ due to a dispersive wave clearly related to the soliton peak near $950 \mathrm{~nm}$. However, it should be noted that experimentally we obtained a peak that is identified as the one related to a dispersive wave at $695 \mathrm{~nm}$. The differences can be related to errors in dispersion characteristics used in simulation calculated based on arbitrary thresholded SEM photo of the fiber.

\section{Conclusions}

The introduction of subwavelength holes in the core of PCFs allows the modification of the fibre dispersion characteristics. The introduction of high-index central subwavelength elements in the core may result in dramatic changes of general parameters of the fiber, where effective mode area of fundamental mode is reduced. In this case the second mode becomes dominant and anomalous dispersion over a long near IR range is obtained. A PCF made of NC21 silicate glass with air-glass cladding and core with subwavelength rod of SF6 glass was fabricated and the successful generation of a supercontinuum in the range $6651055 \mathrm{~nm}$ with a flatness of

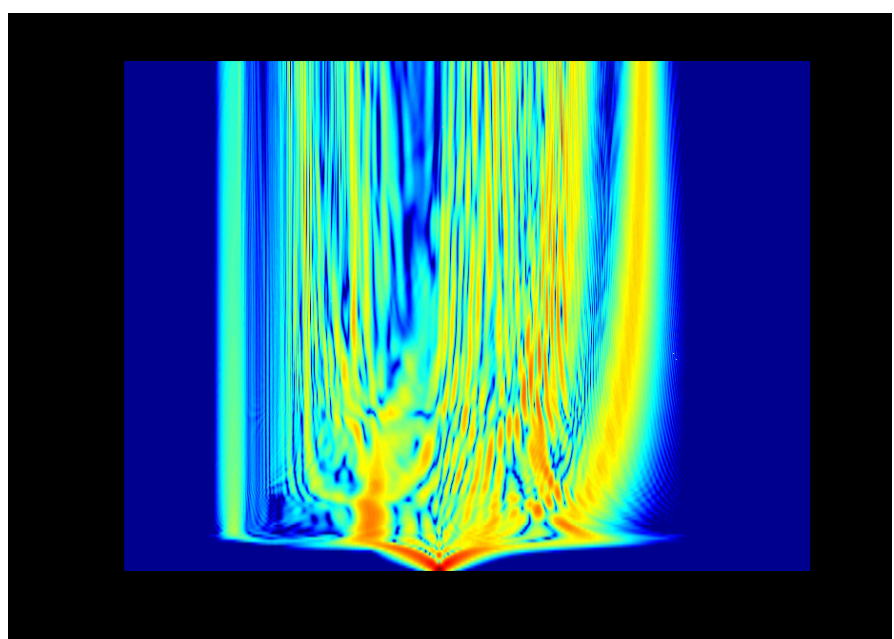

FIG. 11 Simulation of spectrum broadening in $22 \mathrm{~cm}$ long sample of PCF with subwavelength glass structure in the core for pulse energy of $2.6 \mathrm{~nJ}$.

$12 \mathrm{~dB}$ using low energy femtosecond pulses at $807 \mathrm{~nm}$ was observed.

\section{Acknowledgements}

This work is supported by the Polish Ministry of Science and Higher Education research grants NN515 244737 and NN515 523738 .

\section{References}

[1] J. M. Dudley, G. Genty, and S. Coen, "Supercontinuum generation in photonic crystal fiber", Rev. Mod. Phys. 78, 1135-1184 (2006).

[2] R. Buczynski, H. T. Bookey, D. Pysz, R. Stepien, I. Kujawa, J. E. McCarthy, A. J. Waddie, A. K. Kar, and M. R. Taghizadeh, " Supercontinuum generation up to $2.5 \mu \mathrm{m}$ in photonic crystal fiber made of lead-bismuth-galate glass", Laser Phys. Lett. 7, 666-672 (2010).

[3] X. Yu, P. Shum, N. Q. Ngo, W. J. Tong, J. Luo, G. B. Ren, Y. D. Gong, and J. Q. Zhou, "Silica-Based Nanostructure Core Fiber", IEEE Photonic Tech. L. 31, 1480 - 1482, (2007).

[4] A. Wang A. George, J. Liu, J. Knight, "Highly birefringent lamellar core fiber", Opt. Express 13, 5988-5993 (2005).

[5] K. Schuster, J. Kobelke, S. Grimm, A. Schwuchow, J. Kirchhof, H. Bartelt, A. Gebhardt, P. Leproux, V. Couderc, and W. Urbanczyk, "Microstructured fibers with highly nonlinear materials", 0pt. Quant. Electron. 39, 1057-1069 (2007).

[6] K. Saitoh, N. Florous, and M. Koshiba, "Ultra-flattened chromatic dispersion controllability using a defected-core photonic crystal fiber with low confinement losses", Opt. Express 13, 8365-8371 (2005).

[7] B. Kibler, P.-A. Lacourt, F. Courvoisier, and J.M. Dudley, "Soliton spectral tunnelling in photonic crystal fibre with sub-wavelength core defect", Electron Lett. 43, 967-968 (2007).

[8] G. S. Wiederhecker, C. M. B. Cordeiro, F. Couny, F. Benabid, S. A. Maier, J. C. Knight, C. H. B. Cruz, and H. L. Fragnito, "Field enhancement within an optical fibre with a subwavelength air core", Nat. Photonics 1, 115-118 (2007).

[9] Y. Ruan, H. Ebendorff-Heidepriem, S. Afshar, and T. M. Monro, "Light confinement within nanoholes in nanostructured optical fibers", Opt. Express 18, 26018-26026 (2010). 
[10] B.-W. Liu, M.-L. Hu, X.-H. Fang, Y.-F. Li, L. Chai, C.-Y. Wang, W. Tong, J. Luo, A. A. Voronin, A. M. Zheltikov, "Stabilized soliton selffrequency shift and 0.1- $\mathrm{PHz}$ sideband generation in a photoniccrystal fiber with an air-hole-modified core", Opt. Express 16, 14987-14996 (2008).

[11] B. Ung, M. Skorobogatiy, "Chalcogenide microporous fibers for linear and nonlinear applications in the mid-infrared", opt. Express 18, 8647- 8659 (2010).

[12] COMSOL Multiphysics 3.4 (2007) http://www.comsol.com.

[13] D. Lorenc, M. Aranyosiova, R. Buczynski, R. Stepien, I. Bugar, A. Vincze, D. Velic, "Nonlinear refractive index of multicomponent glasses designed for fabrication of photonic crystal fibers", Appl. Phys. B - Lasers 0. 93, 531 (2008).

[14] R. Buczynski, D. Pysz, R. Stepien, A.J. Waddie, I. Kujawa, R. Kasztelanic, M. Franczyk, M.R. Taghizadeh, "Supercontinuum generation in photonic crystal fibers with nanoporous core made of soft glass", Laser Phys. Lett. 8, 443-448 (2011).

[15] S. V. Afshar, W. Q. Zhang, H. Ebendorff-Heidepriem, and T. M. Monro, "Small core optical waveguides are more nonlinear than expected: experimental confirmation", Opt. Lett. 34, 3577-3579 (2009).

[16] J. C. Travers, M. H. Frosz, and J. M. Dudley, "Nonlinear fibre optics overview" (Chap. 3) in Supercontinuum generation in optical fibers, J. M. Dudley and J. R. Taylor, eds. (Cambridge University Press, 2010) 